\title{
A Relationship between Ultrasonic Integrated Backscatter and Myocardial Contractile Function
}

Samuel A. Wickline, Lewis J. Thomas III, James G. Miller, Burton E. Sobel, and Julio E. Perez

Departments of Medicine and Physics, Washington University, St. Louis, Missouri 63110

\begin{abstract}
We have shown previously that the physiologic, mechanical cardiac cycle is associated with a parallel, cardiac cycle-dependent variation of integrated backscatter (IB). However, the mechanisms responsible are not known. The mathematical and physiological considerations explored in the present study suggest that the relationship between backscatter and myocardial contractile function reflects cyclic alterations in myofibrillar elastic parameters, with the juxtaposition of intracellular and extracellular elastic elements that have different intrinsic acoustic impedances providing an appropriately sized scattering interface at the cellular level. Cardiac cycle-dependent changes in the degree of local acoustic impedance mismatch therefore may elicit concomitant changes in backscatter. Because acoustic impedance is determined partly by elastic modulus, changes in local elastic moduli resulting from the non-Hookian behavior of myocardial elastic elements exposed to stretch may alter the extent of impedance mismatch. When cardiac cell mechanical behavior is represented by a three-component Maxwell-type model of muscle mechanics, the systolic decrease in IB that we have observed experimentally is predicted. Our prior observations of regional intramural differences in IB and the dependence of IB on global contractile function are accounted for as well. When the model is tested experimentally by assessing its ability to predict the regional and global behavior of backscatter in response to passive left ventricular distention, good concordance is observed.
\end{abstract}

\section{Introduction}

Myocardial ultrasonic tissue characterization represents a promising noninvasive quantitative approach to the study of cardiac function and structure $(1,2)$. Several potentially clinically applicable ultrasonic approaches have been explored recently to differentiate normal from pathological cardiac conditions. Analysis of data based on gray level images or textural patterns obtained with the use of conventional echocardiography is convenient, but may be limited by the lack of uniform standardization procedures or by operator-dependent modification of the ultrasonic energy transmission and image gain settings. In

Portions of this work were presented at the American Federation for Clinical Research (AFCR) meeting in Washington, DC, 1985, and at the Tenth International Symposium on Ultrasonic Imaging and Tissue Characterization meeting in Washington DC, 1985.

Address all correspondence and reprint requests to Dr. Perez, Cardiovascular Division, Department of Internal Medicine, Washington University School of Medicine, Box 8086, 660 S. Euclid Ave., St. Louis, MO 63110.

Received for publication 24 June 1985

J. Clin. Invest

(C) The American Society for Clinical Investigation, Inc.

$0021-9738 / 85 / 12 / 2151 / 10 \quad \$ 1.00$

Volume 76, December 1985, 2151-2160 our laboratory, quantitative analysis of tissue ultrasonic characteristics has been based on the parameters of backscatter and attenuation. Both measurements are independent of the electronics of the imaging system and easily standardized.

Characterization of myocardium based on frequency averaged, or integrated, backscatter measurements permits differentiation in vitro and in vivo of normal from ischemic or cardiomyopathic tissue in experimental animals (2-7). We have previously demonstrated that the physiologic, mechanical cardiac cycle (contraction and relaxation) is associated with a parallel, cardiac cycle-dependent variation of integrated backscatter (IB) ${ }^{1}$ (8). We recently developed an analog ultrasonic data acquisition system in order to display this cyclic variation of IB in real time, and applied it to open-chest dogs and normal human volunteers (9). With the use of this system, global and regional intramural myocardial contractile function are reflected quantitatively by the rate of change and amplitude of cyclic variation of IB in dogs (10). Although the cyclic variation of IB has been reproduced in other laboratories (11), its determinants have not been elucidated.

This study was designed to develop a physiologically based model for the cyclic variation of backscatter that would account for the relationship between backscatter and myocardial contractile function in terms of myofibrillar elastic characteristics. The model was evaluated by testing its predictions of the behavior of IB in response to passive mechanical distention of the left ventricle in diastolic cardiac arrest with and without perfusion of the myocardium. Our report describes the model and its assumptions, the experimental protocols used to test the response of IB to passive fiber stretching, and the active and passive components of the model developed.

Physiologic considerations underlying the model developed. An association between myocardial contractile function and IB has been reported (10). Global alterations in contractile function inducible in open-chest dogs with either paired pacing (increased) or propranolol (decreased) are associated with concomitant increases or decreases in the maximum rate of change of timevarying IB waveforms that parallel changes in contractile function measured independently by maximum rate of change of left ventricular pressure ( $\left.\mathrm{dP} / \mathrm{dt}_{\max }\right)$. Regional intramural differences in $\mathrm{dIB} / \mathrm{dt}_{\max }$ (maximum rate of change of time varying IB) are characterized by greater maximal rates of change of IB in midmyocardial zones ("distal," with respect to the epicardium) than in subepicardial zones ("proximal") at all levels of global contractile function. ${ }^{2}$ Regional intramural differences are man-

1. Abbreviations used in this paper: $\mathrm{dB}$, decibels; $\mathrm{dP} / \mathrm{dt}_{\max }$, maximum rate of change of left ventricular pressure; $E_{p e}$, elastic modulus of the parallel elastic element; $E_{s e}$, elastic modulus of the series elastic element; IB, integrated backscatter; $L V$, left ventricular; $Z_{e}$, extracellular acoustic impedance; $Z_{i}$, intracellular acoustic impedance.

2. Midmyocardial zones and subepicardial zones will be referred to as "distal" and "proximal," respectively, for the remainder of the paper. 
ifest also as greater amplitudes of cyclic variation of IB in distal compared with proximal regions.

These observed regional differences in backscatter may depend on the systematic variation of sarcomere contractile function and fiber stress throughout the myocardial wall. Diastolic sarcomere length increases progressively from the outer to the inner wall except in the penultimate subendocardium, where length is decreased (12-14). Because of the Frank-Starling law, distal sarcomeres may attain greater velocities of shortening, greater fiber stresses, and/or greater fiber shortening than sarcomeres in proximal regions. Inner wall myocardial segments contract to a greater extent and more rapidly than do outer wall segments (15-17). Subendocardial regions develop greater local tissue pressure at faster rates than do subepicardial regions (18). The transmural gradient in oxygen tension (subepicardial is greater than subendocardial) may reflect the greater functional energy requirements associated with relatively enhanced contractile function of subendocardial sarcomeres (19).

Augmented contractile performance in distal regions theoretically should elicit transmural differences in developed fiber stress, or "regional afterload." Although physical measurements of left ventricular (LV) wall stress may be technically difficult to obtain (20), calculations of transmural wall stress based on mathematical models are consistent with regional variations in fiber contractile performance and developed stress. Streeter first used a geometrically realistic $\mathrm{LV}$ wall stress model to determine that fiber stress and sarcomere shortening increase from outer to inner wall zones, except in the penultimate subendocardial zone, where stress decreases $(21,22)$. More recently, Chadwick (23) published a fiber-continuum wall stress model that incorporates dynamic material elastic parameters and anisotropic myocardial fiber geometry into the calculation of regional intramural stress-strain relationships. His computations, and those of others using different models $(24,25)$, support the concept of transmural gradients for diastolic sarcomere length, extent of sarcomere shortening, and systolic fiber tension with nonlinear increases from epicardium to midwall, and slight decreases in the subendocardium.

Our experimental observations of greater amplitudes and rates of change of IB in distal than in proximal regions (10) are consistent with those models that report greater developed stress and velocity of shortening of mid-to-inner wall fibers. This concordance suggests that the cyclic variation of backscatter may reflect regional variations in the velocity and extent of sarcomere shortening and in developed fiber stress. The close correlation that we have observed between global changes in contractile function and concomitant alterations in the rate of change of backscatter is consistent also with this relationship (10).

$A$ relationship between backscatter and myocardial elastic elements. In a broad sense, ultrasonic interrogation of myocardial tissue characterizes material density and elastic parameters (see Appendix 1 for an illustration). For the general case of scattering produced by inhomogeneities smaller than the insonifying wavelength, the magnitude of ultrasonic backscatter is determined by local differences in material density and stiffness, or elastic modulus. For the specific case of backscatter $\left(180^{\circ}\right.$ scattering), backscattered energy is approximately proportional to local differences in acoustic impedances, i.e., products of the square root of mass density and the appropriate elastic modulus. Thus, backscatter is determined by the extent of local acoustic impedance mismatch between interfacing tissue regions exhibiting different elastic moduli and/or different densities. We hy- pothesize that variations in these physical parameters associated with the physiologic cycle of contraction and relaxation will affect the extent of local acoustic impedance mismatch and hence the magnitude of energy backscattered. Because myofibril contractile function is expressed physiologically through series elastic elements, we propose that the cyclic variation of backscatter reflects local changes in elastic parameters of fibers governed by sarcomere contractile function.

Any of several anatomic elastic structures might establish a scattering interface between regions of different acoustic impedance. Shung and Reid (26) and O'Donnell et al. (27) have demonstrated that myocardial scatterers appear comparable in size to myocytes. Collagen is a primary determinant of backscatter, and backscatter depends not only on the total amount of collagen present in tissue but also on its three-dimensional organization (28). Scanning microscopic studies of Caulfield and Borg (29) demonstrate that an extensive and complex collagen matrix invests the cardiac cell sarcolemma and pervades the interstitial milieu (29). Furthermore, the cell surface is endowed with longitudinally oriented, supporting "surface cables" (30). When the cell membrane and interstitial structures are removed from individual isolated canine cardiac cells, the "skinned" fibers become considerably more compliant to passive stretch (31). Thus, external structural matrices may contribute to myocardial passive stiffness or resistance to stretch.

The intracellular domain is composed of the sarcomere assembly and other organelles that contribute indirectly to function (such as mitochondria and sarcoplasmic reticulum). A complex "intermediate fiber matrix" invests the sarcomeres internally, though it is more difficult to visualize than the external collagen matrix $(32,33)$. This matrix interconnects Z-discs, M-bands, intercalated discs, sarcoplasmic reticulum, and sarcolemma. Thus, it appears to provide load-bearing cytoskeletal support and to maintain sarcomere register during fiber contraction. Other sarcomere-associated elastic fibers appear to connect thick filaments to Z-discs (34). The presence of these structurally different intracellular and extracellular elastic domains (represented in Fig. 1) suggests that the juxtaposition of intracellular and interstitial-sarcolemmal elastic elements may constitute regions

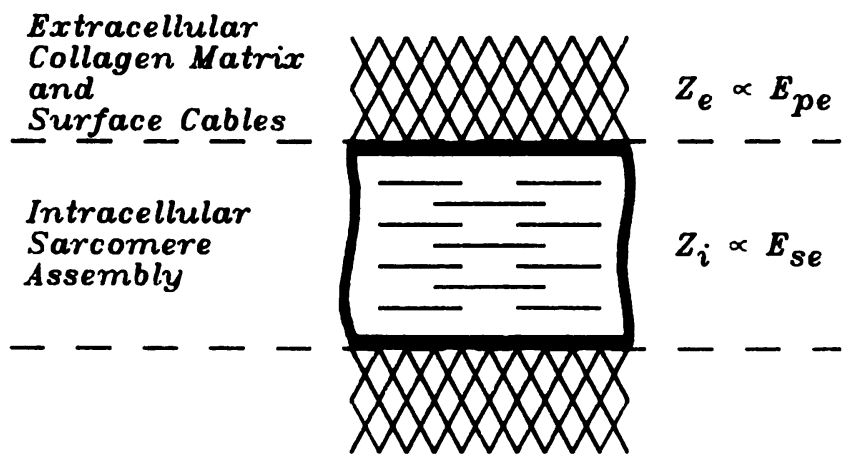

Figure 1. Proposed anatomic structure for myocardial scatterers. Refer to text for full explanation. Myocardial scatterers are approximately the size of myocytes. The $\mathrm{Z}_{e}$ is proportional to the appropriate $\mathrm{E}_{\mathrm{pe}}$ and is identified with the extracellular collagen matrix and surface cables. The $Z_{i}$ is proportional to the appropriate $E_{s e}$ and is identified with the intracellular sarcomere assembly and associated intracellular elastic fibers. If $\mathrm{Z}_{e}$ differs from $\mathrm{Z}_{i}$ at base line, then the juxtaposition of extracellular and intracellular elastic domains forms a scattering interface comprised by elastic elements with different base-line acoustic impedances. 
of local acoustic impedance mismatch. The absolute basal impedances need differ only modestly at the scattering interface in view of the "relatively" poor scattering observed from myocardial tissue (50-60 dB below that from a stainless-steel reflector).

Description of the model developed. Because it is not feasible to directly measure regional differences in acoustic impedance in the beating heart, we formulated a hypothesis about the relationship between intracellular and extracellular acoustic impedance $\left(Z_{i}\right.$ and $Z_{e}$, respectively) and evaluated its predictive accuracy under various experimental conditions. We propose that the cardiac cycle-dependent variation of backscatter results from cyclic variation in local acoustic impedance mismatch as a consequence of relative changes in elastic modulus and/or density in interfacing elastic domains. If cardiac cell mechanical behavior is depicted in terms of a simple Maxwell-type model (Fig. 2), then the activated contractile element stretches a series elastic element during "cell-isometric" tension development in systole, until the fiber tension matches the regional afterload (35). Cardiac cells then shorten during the ejection phase in the intact heart with further stretching of the series elastic element dependent on subsequent alterations in regional afterload. Because the series elastic element exhibits non-Hookian elastic behavior with stretch $(36,37)$, its stiffness, or elastic modulus $\left(E_{s e}\right)$, increases with progressive elongation until regional afterload is matched. Because the series elastic element represents a subset of the mutable intracellular elastic domain, intracellular stiffness and acoustic impedance would be expected to increase.

Physiological changes in the extracellular elastic domain associated with contraction are quite different. This region comprises the subset of passive load-bearing structures, or parallel elastic elements and the interspersed microvascular network. The parallel elastic element exhibits non-Hookian elastic behavior because its stiffness increases with passive diastolic fiber stretching (38). The Maxwell muscle model specifies that parallel elastic element length remains constant during cell-isometric series elastic element stretching but then decreases as the cell shortens during the ejection phase of systole in the intact heart. Because the parallel elastic element is fixed during the phase of

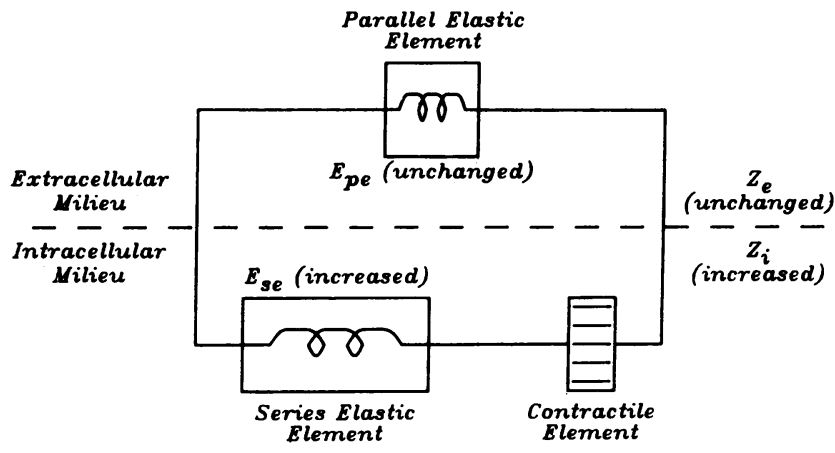

Figure 2. Proposed explanation for the decrease of ultrasonic IB under the condition of physiologic contraction. Symbols are the same as those in Fig. 1. A Maxwell-type model for the mechanical behavior of myocardial muscle is assumed. As the contractile element shortens, the series elastic element is stretched and the $Z_{i}$ increases relative to the $Z_{e}$. The parallel elastic element is fixed. Assume that base-line $Z_{e}$ is different from and greater than $Z_{i}$. Then, as $Z_{i}$ increases relative to $Z_{e}$, the acoustic impedance mismatch between $Z_{i}$ and $Z_{e}$ decreases and IB also decreases. Backscatter subsequently increases with relaxation as the acoustic impedance mismatch is restored. initial series elastic element stretching and then relaxes during the phase of cell shortening, the extracellular elastic modulus $\left(E_{p e}\right)$ and $Z_{e}$ would remain constant or be expected to decrease. The $Z_{i}$ will increase relative to the extracellular impedance during systole, and the local acoustic impedance mismatch will change. Accordingly, backscatter will change progressively during systole and return to base line during diastole.

Although this mechanism would account for a cyclic variation in backscatter, we must consider the question of why IB decreases during systole. As will be shown below, basal $Z_{e}$ exceeds $Z_{i}$. This relation appears reasonable in view of the extracellular abundance and complex arrangement of collagen that is a primary determinant of backscatter. If a relative increase in $Z_{i}$ occurs in systole when the series elastic element is stretched, then $Z_{i}$ approaches $Z_{e}$ and the local acoustic impedance mismatch decreases. Therefore, the magnitude of energy backscattered from this diminished scattering interface would be expected to decrease during systole.

Our previous observations regarding the effects of global and regional differences in contractile performance on IB can be reexamined in the context of these considerations (10). Global alterations in contractile function with paired pacing or propranolol will increase or decrease the rate at which the acoustic impedance mismatch changes, and should therefore similarly affect the rate of change of IB. Regional intramural differences in IB reflect the greater fiber shortening velocities and stresses in distal than in proximal regions as a consequence of augmented contractile element performance and greater series elastic element stretch. Distal series elastic element stiffness, or elastic modulus, will change relatively more than proximal series elastic element elastic modulus during contraction. Therefore, $Z_{i}$ is expected to approach $Z_{e}$ more closely in distal than in the proximal regions, and the distal acoustic impedance mismatch is expected to decrease to a greater extent. At end-systole, less backscatter will occur at the relatively poorer distal scattering interface than at the better proximal scattering interface. A greater cyclic decrease of IB in distal regions will result. Because the distal series elastic element is stretched to a greater extent than the proximal series elastic element in the same interval (by end-systole), the local acoustic impedance mismatch decreases more rapidly in the distal region. Therefore, the rate of change of distal backscatter is expected to be greater.

Predictions of the model. With the use of the model outlined above, we should be able to predict the backscatter response to passive myocardial fiber stretching. The physiologic events associated with passive fiber stretching according to the Maxwelltype muscle model are represented in Fig. 3. If we assume that the contractile element extends freely as the fiber is stretched passively, then stress and strain on the series elastic element are relieved. Thus, this element exhibits constant stiffness (or $E_{s e}$ ). However, the parallel elastic element supports passive fiber stress, and its stiffness (or $\mathrm{E}_{\mathrm{pe}}$ ) increases with progressive stretch. Recalling the initial assumption of greater extracellular than intracellular acoustic impedance $\left(Z_{e}\right.$ is greater than $\left.Z_{i}\right)$ and the dependence of acoustic impedance on elastic modulus, we note that $Z_{e}$ increases relative to $Z_{i}$. The acoustic impedance mismatch now increases at the scattering interface. Therefore, the model predicts an increase in backscatter with passive fiber stretching.

Two other considerations were addressed in testing this model. Computations by several investigators suggest that regional intramural differences in diastolic wall stress characterize subendocardial, midmyocardial, and subepicardial regions (re- 


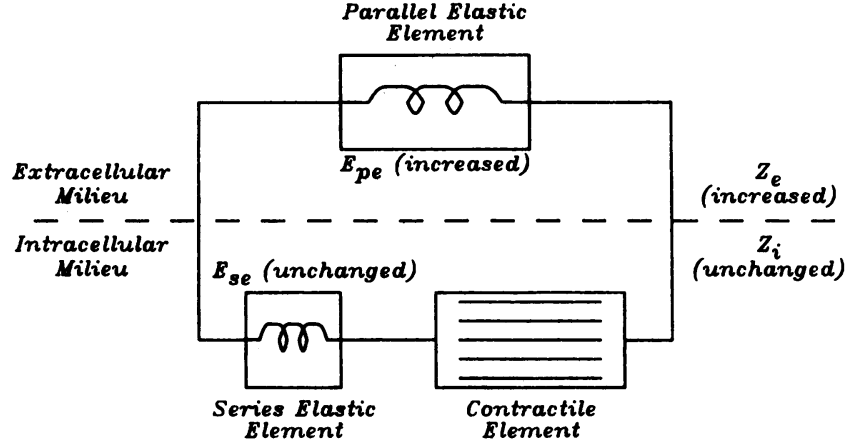

Figure 3. Prediction for the behavior of ultrasonic IB under the condition of passive LV distention. Format is the same as in Fig. 2. As the parallel elastic element is stretched, the contractile element extends freely and $Z_{e}$ increases relative to $Z_{i}$. The series elastic element is fixed. Assume that base-line $Z_{e}$ is greater than $Z_{i}$ as in the case of physiologic contraction (Fig. 2). Then, as $Z_{e}$ increases relative to $Z_{i}$, the acoustic impedance mismatch between $Z_{e}$ and $Z_{i}$ increases and IB also increases. Backscatter subsequently decreases with relaxation as the acoustic impedance mismatch decreases.

viewed by Yin in reference 39). Most computations support a nonlinear increase in diastolic circumferential wall stress from subepicardial to subendocardial zones. To evaluate this possibility in terms of the model developed here, the cyclic variation of IB was measured in both distal and proximal intramural regions during passive mechanical LV distention under the condition of diastolic cardiac arrest.

In addition, the potential contribution of myocardial blood flow to the cyclic variation of backscatter was evaluated. Although we have previously demonstrated that the intravascular presence or absence of formed blood elements may affect the static determination of time-averaged backscatter (3), it is not clear what role blood flow or intravascular volume plays in the dynamic cardiac-cycle dependent variation of backscatter. Therefore, the issues of whether vascular compressive effects and/or phasic variations in blood flow represent sources of cyclic variation were addressed by measuring backscatter with and without coronary perfusion during passive mechanical ventricular distention. We attempted to generate vascular compressive effects similar to those that might occur with systolic ventricular contraction by using a wide range of ventricular distending pressures under conditions of diastolic cardiac arrest. This approach served to dissociate the passive effects of hydrostatic vascular compression from the active mechanical events associated with ventricular systole. By discontinuing coronary flow abruptly and rapidly measuring backscatter under the same conditions as above, we sought to determine the consequences of a lack of phasic variation in blood flow and intravascular volume on the cyclic variation of backscatter.

\section{Methods}

The overall experimental approach comprised three sequential protocols. First, base-line physiologic cycle variation was measured in paced openchest dogs in proximal and distal intramyocardial regions. Then, the dogs were placed on ventricular bypass under conditions of end-diastolic cardiac arrest and the left ventricle was distended periodically with an intracavitary balloon while cyclic variation was again measured. For the final protocol, bypass and coronary flow were discontinued abruptly, but periodic mechanical distention maintained, and cyclic variation was measured again rapidly.
The protocols were designed to minimize several potentially confounding problems. The assumption of free extension of the contractile element with passive distention necessitated maintenance of tissue viability and prevention of significant rigor-complex formation. Accordingly, ventricular diastolic arrest was induced in dogs with aortic root injections of saturated potassium chloride, and the coronary arteries were perfused at physiologic pressures, temperatures, and oxygen concentrations by establishing venoarterial cardiac bypass. The occurrence of stress-relaxation and creep with distention might also have affected static determinations of IB as a consequence of time-dependent tissue viscoelastic changes $(38,40)$. Therefore, measurements of dynamic alterations in backscatter were made under conditions of cyclic LV distention induced with an intracavitary balloon.

Surgical preparation. Mongrel dogs (15-30 kg) were anesthetized with intravenous pentobarbital $(20-30 \mathrm{mg} / \mathrm{kg})$ and ventilated with room air supplemented with oxygen to maintain physiologic concentrations of arterial blood gases. A femoral artery cannula was inserted to monitor aortic pressure and blood gases. A right thoracotomy was performed. The atrioventricular node was injected with $1-2 \mathrm{ml}$ of formalin to induce complete heart block. The heart was paced from the right ventricle at a rate of 100 beats $/ \mathrm{min}$. The right thoracotomy was closed and a left thoracotomy performed to expose the LV free wall. A fluid-filled cannula was inserted into the $\mathrm{LV}$ cavity through the apex to monitor $\mathrm{LV}$ pressure. A $2 \times 2-\mathrm{cm}$ region of the anterior free wall was demarcated $\sim 2 / 3$ down from the circumflex artery toward the apex, and ten sites were specified for ultrasonic data collection. Base-line measurements of IB were made in proximal and distal intramural regions at each of the 10 sites.

Ventricular bypass was established subsequently as follows (Fig. 4). Dogs were anticoagulated with intravenous injections of heparin sulfate $(10,000 \mathrm{U})$. Venous return was collected by cannulating the right ventricle with a 24-French Bardic catheter and blood drained through silastic tubing by gravity into a pediatric-sized bubble oxygenator. Oxygen flow rates (5-10 liters/min of $100 \% \mathrm{O}_{2}$ ) were adjusted to provide partial pressures of $100-200 \mathrm{mmHg}$. The temperature of the blood was maintained at $37^{\circ} \mathrm{C}$. A roller pump circulated blood through a filter with a particle exclusion size of $40 \mu \mathrm{m}$. The aortic root was perfused with a 22-French Bardic cannula through the right brachiocephalic artery. Mean perfusion

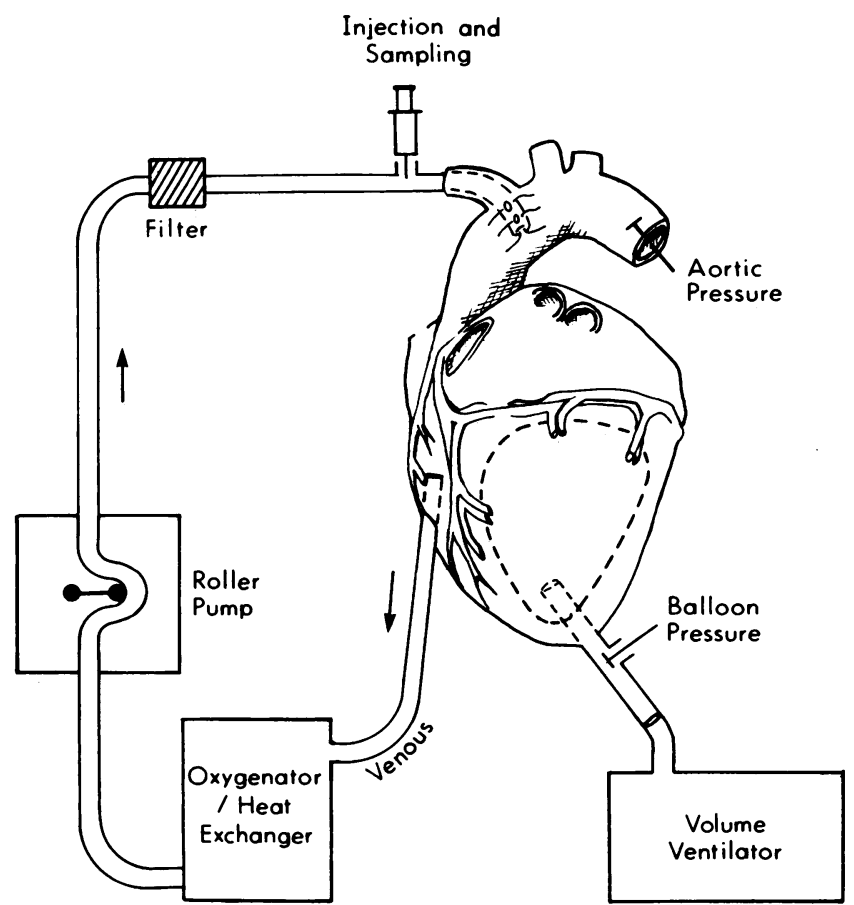

Figure 4. Experimental set-up for passive mechanical LV distention under the condition of end-diastolic cardiac arrest. Refer to text for details. 
pressure was $79 \pm 23 \mathrm{mmHg}$. The system was primed with 1 liter of a modified Krebs-Henseleit solution containing 3\% hydroxyethyl starch (Hespan). Saturated potassium chloride was added to the priming solution and injected in boluses through the perfusing cannula to maintain diastolic cardiac arrest.

The LV apical pressure catheter was replaced by a specially constructed flexible latex balloon mounted at the tip of a 26-French Bardic catheter. The balloon was inflated 30 times/min with a volume respirator to generate a wide range of filling pressures. Filling pressures were measured with a strain gauge transducer through a port located near the catheter tip. The time required to establish the stable cardiac bypass preparation was $\sim 10 \mathrm{~min}$

IB was measured in proximal and distal regions at five of the ten sites previously demarcated. Arterial perfusion was then interrupted while passive cyclic LV distention was continued with the previous filling volume. Backscatter was recorded again from the same sites and regions within 5-10 min of stopping perfusion.

Collection of ultrasonic data. Our procedure for measuring IB in real time has been described previously $(9,10)$. In brief, a $5-\mathrm{MHz}$ focused piezoelectric transducer was used to transmit and receive broadband ultrasonic signals at a pulse rate of $1 \mathrm{kHz}$ in a direction normal to the epicardial surface. Two 3- $\mu$ s portions of the backscattered signal were gated from two contiguous $2.25-\mathrm{mm}$ intramyocardial regions (proximal and distal), with front boundaries located 2 and $4.25 \mathrm{~mm}$ below the initial epicardial specular reflection. The use of these particular gates maximized the separation between discrete subepicardial and midwall locations while avoiding confounding specular echoes. Gated signals were fed into an acoustoelectric energy detector that produced a peak output voltage directly proportional to the average energy contained in the signal over the frequency band of interest. Output voltages representing IB at each instant in the heart cycle were referenced to the voltage produced by backscatter from a steel reflector and converted on-line to decibels (dB). The time-varying IB values (in units of " $\mathrm{dB}$ down from a perfect reflector") were displayed in real time on a standard 2D/M-mode imager screen to visualize the cyclic variation of IB previously reported for normal hearts $(6,8)$. This periodic IB signal was passed to a lock-in analyzer (Dynatrac 3; Ithaco, Inc., Ithaca, NY) and resolved to its fundamental frequency (for a period of one heart cycle) with in-phase and out-ofphase components relative to the LV pressure waveform. The lock-in output was processed further on-line to produce a real time display of two parameters: (1) IB signal phase relative to the LV pressure waveform; and (2) the amplitude of the cyclic variation of IB. With the use of a 4-s lock-in time constant, $\sim 7$ heart beats were incorporated for each analysis of phase and amplitude. When backscatter was measured under conditions of physiologic contraction, the LV pressure waveform was used as the lock-in reference. When IB was measured under conditions of passive mechanical LV cyclic distention, the balloon-filling pressure waveform served as the reference.

Analysis. The phases and the amplitudes of cyclic variation of IB determined in real time at single sites were averaged for discrete intramural regions in individual dogs to yield regional phase and amplitude data. This facilitated a more conservative statistical analysis on a dog-by-dog rather than site-by-site basis. Mutually independent comparisons were planned a priori to assess the differences between (1) cyclic variation for proximal vs. distal regions under conditions of physiologic contraction, (2) cyclic variation for proximal vs. distal regions under conditions of passive LV distention, and (3) cyclic variation for perfused vs. nonperfused LV distentions (in both regions). Paired $t$ tests were used to assess the significance of differences (41). Data are reported as mean \pm SD.

\section{Results}

Physialogic contraction and relaxation. Representative snapshots of actual time-varying IB waveforms are illustrated in Fig. 5 for physiologic contraction and passive mechanical LV distention. IB clearly decreases in systole with physiologic contractions, but increases with passive LV distention. Amplitudes of

\section{PHYSIOLOGIC CONTRACTION}

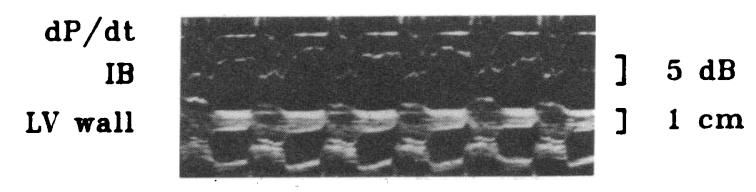

PASSIVE DISTENTION

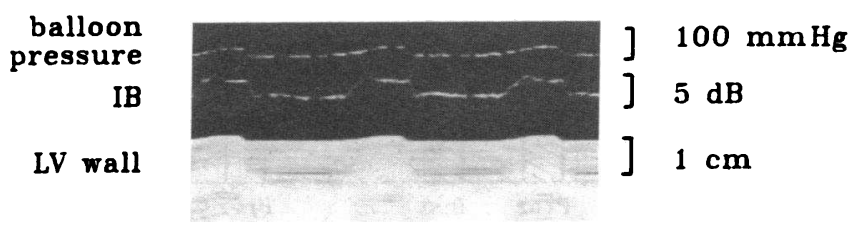

Figure 5. Illustration of actual time-varying IB signals with physiologic contraction (top) and passive mechanical LV distention (bottom). Top: IB decreases in systole and returns to base line in diastole (compare $\mathrm{LV} \mathrm{dP/dt} \mathrm{signal).} \mathrm{Although} \mathrm{the} \mathrm{LV} \mathrm{dP/dt}$ signal is shown here, the LV pressure signal was used as the reference signal for the lock-in detector (see text for details). Bottom: IB increases as the LV intracavitary balloon is filled, and decreases as the balloon relaxes (compare balloonfilling pressure signal). The intracavitary balloon can be visualized just below the $\mathrm{LV}$ wall.

cyclic variation of IB and phase under conditions of physiologic contraction and passive mechanical LV distention in seven dogs are shown in Fig. 6. In the top panel (left), proximal and distal amplitudes for physiologic contractions are compared. Data from 10 sites in proximal or distal regions was averaged to obtain the regional amplitude and phase for a single animal. Distal regions exhibited significantly greater amplitudes of cyclic variation than did proximal regions for normal contractions $(6.72 \pm 0.92$ vs. $5.72 \pm 0.89 \mathrm{~dB} ; P<0.05$ by paired $t$ test). These data are consistent with our previous results demonstrating a $1.5-\mathrm{dB}$ difference in intramural regional amplitude of cyclic variation by analysis offline (10). The bottom panel (left) depicts the frequency of occurrence of phases in the same seven dogs for combined proximal and distal regions. A single occurrence of a phase (i.e., a bar of unit length) represents the average of phases from 10 sites in one region for a single animal. Phases are grouped in ranges of $10^{\circ}$ in the histogram. The mean phase for physiologic contraction was $124 \pm 11^{\circ}$, consistent with our previous results (10). A graphic illustration of normal phase relationships is shown in Fig. 7 (top). The $L V$ pressure waveform is represented as a square wave with a period of one heart cycle. The IB signal is resolved to its fundamental frequency and manifests systolic decreases and diastolic increases in amplitude. The relative phase shift is denoted by the earlier appearance of the IB signal peak.

Passive distention of perfused myocardium. Amplitudes and phases of cyclic variation for passive mechanical LV distention are shown in Fig. 6. In the top panel (right), proximal and distal average amplitudes from five sites in each of seven animals are compared. Distal regions exhibited greater cyclic increases in IB than did proximal regions $(4.83 \pm 1.6$ vs. $3.3 \pm 1.1 \mathrm{~dB} ; P<0.001$ by paired $t$ test). Backscatter was measured at more than one filling pressure in several dogs. The range of filling pressures $(17-92 \mathrm{mmHg}$ ) was extended beyond typical diastolic levels in order to ascertain the vascular compressive effects of large passive hydrostatic pressure gradients on the cyclic variation of backscatter. If the transmural hydrostatic pressure gradient generated during contraction was responsible for the systolic decrease of 

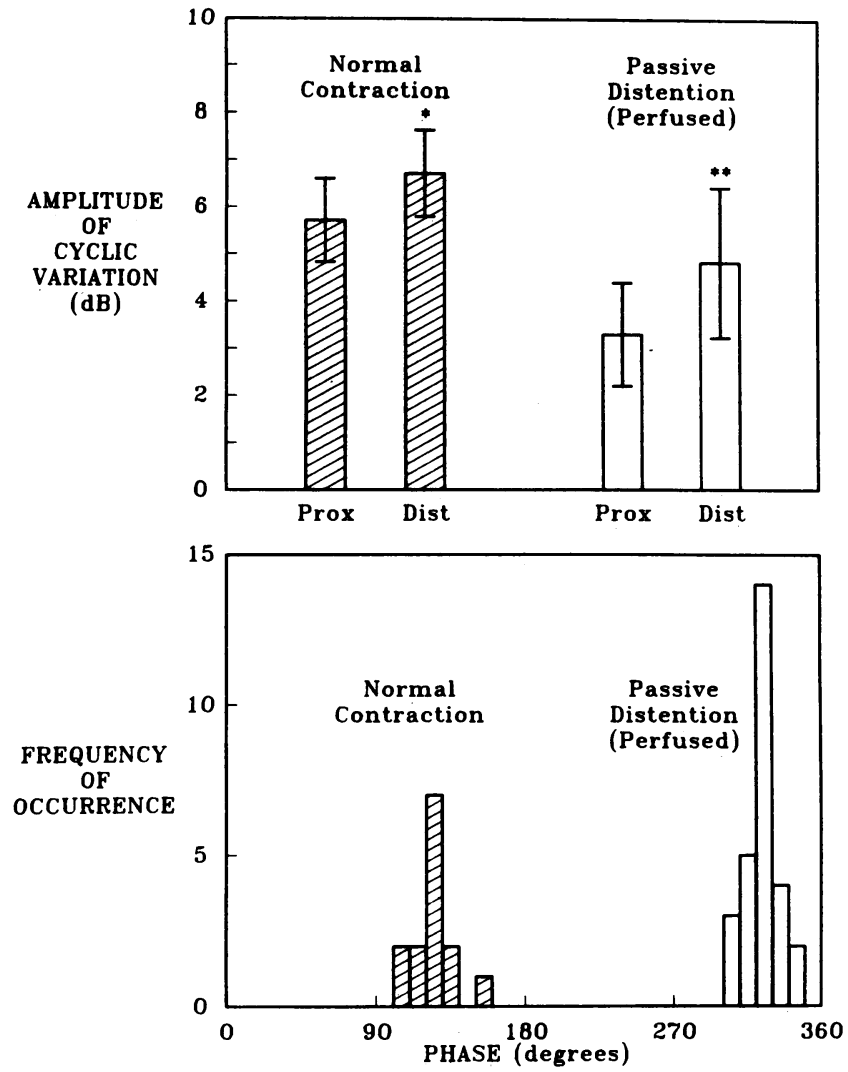

Figure 6. Top: Amplitude of cyclic variation of IB for physiologic contractions (left) and perfused passive LV distentions (right) in proximal (Prox) and distal (Dist) intramural regions. Distal cyclic variations exceed proximal variations for both conditions $\left(^{*}=P<0.05\right.$, and ${ }^{* *}$ $=P<0.001$ for Prox vs. Dist; $n=7$ for each condition). Bottom: Histogram of phases of IB waveforms for physiologic contractions (left) and perfused passive LV distentions (right). Proximal and distal phases are combined for each condition. Each bar width represents $10^{\circ}$. A bar of unit height would represent the average phase for 10 sites in a proximal or a distal region for a single animal under physiologic contraction and for five sites for a single animal under passive distention. The overall average phase for physiologic contractions was $124 \pm 11^{\circ}($ mean $\pm \mathrm{SD})$ and $323 \pm 10^{\circ}$ for passive distentions.

IB, then passive cyclic LV distention to similar cavity pressures should cause similar cyclic decreases of IB.

The phases for passive distention shown in the bottom panel of Fig. 6 (right) were reversed with respect to the phases shown in Fig. 6 for physiologic contraction (left), indicating that integrated backscatter increases as the ventricle is passively filled. The phase reversal of IB cyclic variation is displayed graphically in the bottom panel of Fig. 7. The LV cavity pressure generated by passive filling is represented as a square wave under these experimental conditions. Backscatter is shown to increase as LVP increases, and decrease as the ventricle relaxes. The observation of a phase difference in the cyclic variation of IB between active and passive conditions implies that the transmural hydrostatic pressure gradient, directed from endocardium to epicardium under both conditions, is not responsible fundamentally for the cyclic variation of IB.

Passive distention of nonperfused myocardium. Amplitudes and phases of cyclic variation under conditions of either perfusion or nonperfusion coupled with mechanical LV distention
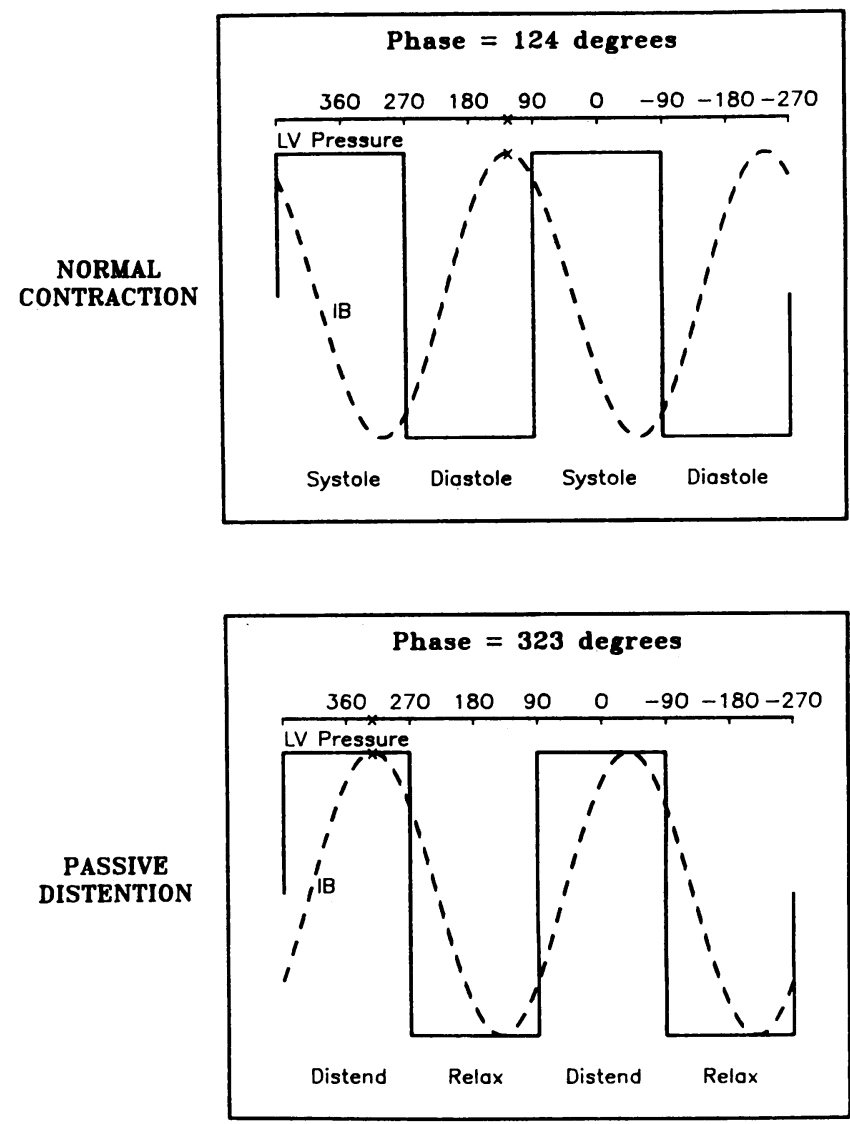

Figure 7. Graphic illustration of the phase relationship between LV pressure and IB waveforms. LV pressure is represented as a square wave and IB as a sine wave. Top: IB decreases in systole with physiologic contraction, and is shifted $124^{\circ}$ relative to the LV pressure waveform ( $\times$ marks the relative shift). Bottom: IB increases with passive distention, and is shifted $323^{\circ}$ relative to the $L V$ pressure waveform.

are shown in Fig. 8. In the top panel, proximal and distal average amplitudes from five sites in seven animals are combined. Amplitudes for perfused (left) and nonperfused (right) states do not differ significantly. The phases under the two conditions are similar as well, indicating that IB increases with passive filling under both sets of conditions (bottom panel, Fig. 8). These measurements were acquired rapidly enough to discount any potentially confounding effects associated with the development of significant tissue edema $(42,43)$. Thus, myocardial perfusion does not affect the cyclic variation of IB in this experimental preparation. Furthermore, neither isolated vascular compressive effects nor phasic alterations in blood flow and/or vascular volume appear to contribute significantly to the phenomenon of cyclic variation.

\section{Discussion}

The model developed to account for the behavior of backscatter based on myocardial tissue elastic parameters relies upon the work of many in the fields of ultrasonic tissue characterization and muscle physiology. Assumptions involving the physical aspects of the relationship between tissue acoustic and elastic properties are detailed in Appendix 1. Selected physiologic features are considered below. 

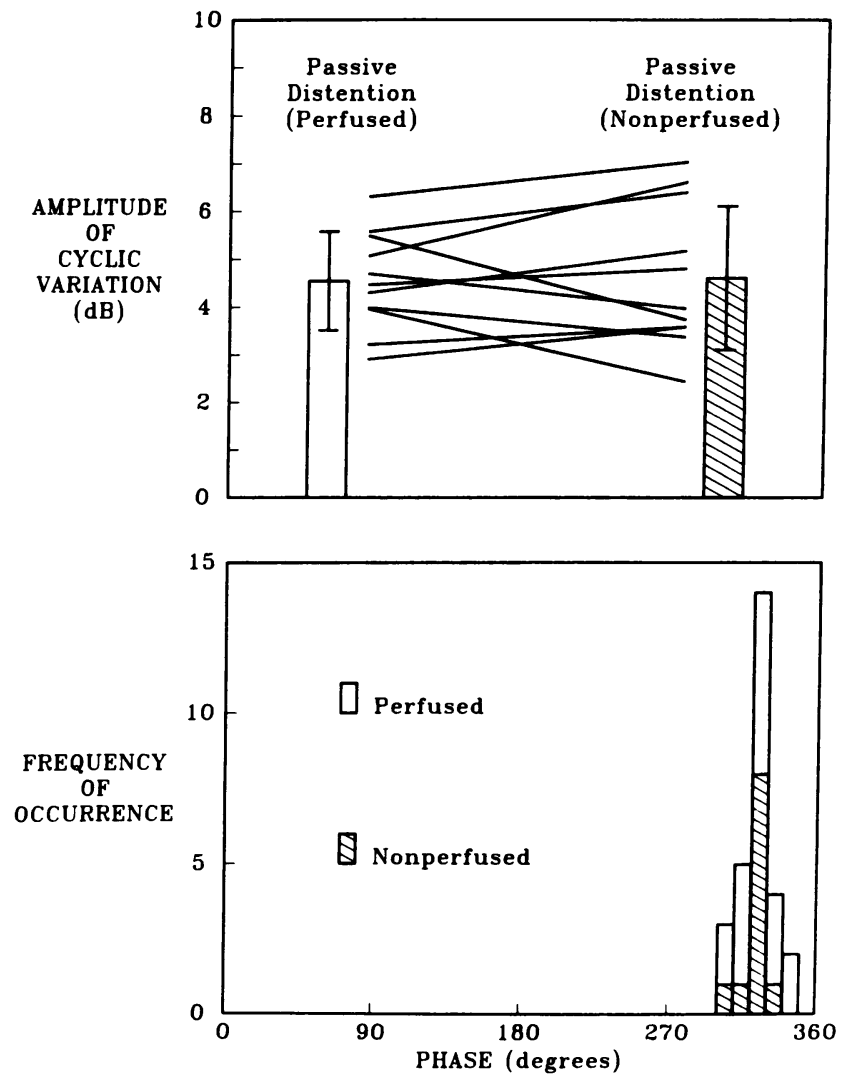

Figure 8. Top: Amplitude of cyclic variation of IB for perfused (left) and nonperfused (right) passive LV distentions. Each line represents the change in the average amplitude of five sites in either a proximal or distal region for one animal after the discontinuation of aortic root perfusion. No significant difference between the two conditions was observed. Bottom: Histogram of phases of IB waveforms for nonperfused passive LV distentions. Format is similar to the bottom panel of Fig. 5. The overall average phase for the nonperfused state was $322 \pm 6^{\circ}$ not different from the average phase for perfused passive distention $\left(323 \pm 10^{\circ}\right)$ as indicated by the overlap of phases.

The experimental observation that backscatter decreases with contraction can be explained by a decrease in local acoustic impedance mismatch at the scattering interface (Fig. 2) if baseline acoustic impedance is greater for the extracellular compared with the intracellular milieu. The observed increase in backscatter with $\mathrm{LV}$ distention requires the same assumption to satisfy the model prediction for passive IB behavior (Fig. 3). Because the predictions are consistent with observations under diverse experimental conditions, and because of appropriate structural characteristics of the intracellular and extracellular domains, the assumption appears to be valid.

The observation of regional intramural differences in backscatter with passive distention is accounted for by the model if greater circumferential fiber stresses prevail in inner wall than in outer wall regions under conditions of passive diastolic filling (39). If this assumption is fulfilled, the distal parallel elastic element would be stretched more and become stiffer than the proximal parallel elastic element (distal $\mathrm{E}_{\mathrm{pe}}$ is greater than proximal $\left.E_{p e}\right)$. At the same time, the intracellular $E_{s e}$ would remain constant in both regions because the contractile element is freely extendible. Thus, the acoustic impedance mismatch will increase more in distal than in proximal regions by end-distention, and the cyclic variation of distal IB will be greater. These observed regional intramural differences in IB concur with expectations of greater inner wall fiber stress based on calculated transmural stress-strain relationships $(23,24,44-46)$.

We have proposed that the local acoustic impedance mismatch reflects the anatomic arrangement of tissue elastic elements in concert with an arrangement of series and parallel elastic elements compatible with that assumed in the Maxwell-type model. Brady has evaluated such configurations in isolated rabbit papillary muscles and determined that no arrangement offers an entirely satisfactory representation of papillary muscle elastic properties (37). Nevertheless, our observations are consistent with the "major effects" that might arise from alterations of elastic parameters in the Maxwell configuration. Furthermore, we are dealing with a more complex system of muscle fibers, with three-dimensional stress-strain interdependencies that may differ considerably from those of the uniaxially loaded papillary muscle system.

We might speculate as to the compatibility of these data with a Voigt-type model, which entails a contractile element and a parallel elastic element, both in series with a series elastic element (35). For the case of active contraction, backscatter should decrease in systole, as predicted for the Maxwell model. The contractile element would stretch the series elastic element, and the parallel elastic element either would not change or else relax. Thus, series elastic element stiffness would increase relative to parallel elastic element stiffness, and elicit a decrease in the local acoustic impedance mismatch and a decrease in backscatter. For the case of passive stretch however, a prediction would be uncertain. Because the parallel elastic element and series elastic element are aligned in series in the Voigt model, we could not determine the effect of stretching the parallel elastic element independent of the series elastic element, as is the case for the Maxwell model. One would have to account for both the viscous and elastic behavior of each element independently in order to estimate the relative change in stiffness and the effect of passive stretch on the local acoustic impedance mismatch. A more complicated protocol for testing the prediction would also be required, involving quick stretches and releases of activated muscle (37). Therefore, further evaluation may be required in order to establish the most appropriate model for the interpretation of backscatter behavior.

Other pertinent considerations relate to tissue ultrasonic anisotropy and attenuation. Measurements of attenuation and backscatter may vary according to the direction of insonification of fibers (47). In our study, the insonifying angle was maintained normal to the epicardial surface within the limits achievable in order to simplify the interpretation of results. We cannot exclude with certainty some systematic geometric contribution of changing fiber angle or shape to the cyclic variation of IB. However, we have previously observed that IB changes rapidly during the isovolumic phase of contraction (10). In contrast, wall thickening and segment length change are minimal during this interval (48).

Because major changes in backscatter appear to precede major changes in fiber dimension that occur during the ejection period, the sliding of myofilaments probably contributes less to the cyclic variation of IB than does the change in elastic modulus. Nevertheless, the possibility that backscatter manifests alterations in the extent of filament overlap may be consistent with this model. Changes in acoustic impedance can occur as a result of changes in either elastic modulus or density. If we hypothesize 
that sliding of filaments produces small local intracellular increments in density, then the $Z_{i}$ will increase relative to $Z_{e}$. Therefore, the impedance mismatch between intracellular and extracellular domains will decrease during systole and elicit the reduction in IB that has been observed experimentally.

Cyclic variations in myocardial tissue attenuation could influence backscatter measurements. A simple estimate indicates that the attenuation coefficient would have to increase to a value of 4-5 times that of base-line attenuation to represent the sole mechanism for the cyclic variation of IB (see Appendix 2 for calculations). Although such changes are improbable, we cannot exclude some impact on IB of alterations in attenuation. The smaller differences in amplitude of cyclic variation of IB in proximal compared with distal regions $(1-1.5 \mathrm{~dB})$ might reflect cyclic changes in attenuation. However, the attenuation coefficient would have to increase to a value of 2.5 times that of baseline attenuation to account for the differences observed-a change that appears to be unlikely.

Predictions and considerations derived from the model developed are pertinent to measurements of backscatter in other tissues. In isolated frog skeletal muscle, IB increases with highly afterloaded auxotonic contraction (49) in contrast with the decrease observed with contraction in cardiac muscle. Simple geometric rearrangements of scatterers or fibers that might result from the sliding of actin and myosin filaments may not be responsible for the changes in IB, since sarcomere contractile function is similar in both systems. Furthermore, little change in sarcomere dimension would be expected with these skeletal muscle contractions. The increase in IB with contraction in the frog muscle may represent an alteration in local acoustic impedance mismatch. Parallel elastic elements of skeletal muscle are more compliant (smaller elastic modulus) than those of cardiac muscle (38). In contrast, series elastic elements of skeletal muscle may be stiffer than those of cardiac muscle (35). A greater relative contribution of actin-myosin cross-bridges to the series elastic element of skeletal muscle cells $(50,51)$ may contribute to this difference. In view of these considerations, we propose that the base-line intracellular acoustic impedance exceeds extracellular impedance for skeletal muscle, which is opposite to the case in cardiac muscle. Thus, with active contraction, the $\mathrm{E}_{\mathrm{se}}$ will increase relative to the $\mathrm{E}_{\mathrm{pe}}$ (see Fig. 2 for comparison), but the acoustic impedance mismatch will now increase at the scattering interface. Therefore, the model developed predicts an increase in backscatter with contraction in frog skeletal muscle. Because the observed changes in IB in frog skeletal muscle occurred in isolated contracting tissue devoid of blood flow and transmuscular hydrostatic pressure gradients (49), local variations in elastic moduli may provide a reasonable explanation of the results.

We have proposed and tested a physiologically based model that accounts to a considerable extent for the behavior of backscatter based on myocardial elastic parameters. It predicts the decrease of IB that occurs with active myocardial contraction and the increase that occurs with passive LV distention. It accounts for the intramural differences between proximal and distal IB measurements in terms of intrinsic regional variations in contractile function and developed fiber stress. It accounts for the response of IB to global alterations in contractile function. Accordingly, the model and its subsequent refinements should enhance the utility of ultrasonic backscatter as a measure of fundamental physiologic processes related to myocardial contractile function.

\section{Appendix 1}

In this section we illustrate the dependence of backscatter on local variations in acoustic impedance for scatterers that are smaller than a wavelength of sound. Although backscatter is typically represented as a function of material density and compressibility, we review an approach that relates backscatter to local variations in acoustic impedance and hence to elastic modulus and density.

For materials such as soft tissue that exhibit elastic properties essentially like those of a liquid, elastic modulus is the reciprocal of compressibility. In the following discussion, we shall use compressibility to describe the elastic characteristics of materials. In order to simplify the formal approach, we shall discuss scattering from a spherical scatterer. Although scattering from a collection of scatterers requires a more sophisticated approach, the objective of this discussion is simply to illustrate a useful variable substitution (given in Eqs. 5 and 6) that applies to any scattering formalism in which the scatterers exhibit only small variations in acoustic properties from those of the surrounding medium.

For an incident plane wave $\left(\Phi_{\text {inc }}\right)$ at large distances from a spherical scatterer, the asymptotic behavior of the pressure wave may be written (52):

$\Phi=\Phi_{i n c}+\Phi_{\text {scat }}=e^{i \dot{k} \cdot \dot{r}}+f(\theta)\left(e^{i k \eta}\right) /(r)$,

where $\vec{r}$ is the distance from the scatterer to a measurement point, and $\vec{k}$ is the wave vector for the incident plane wave. In the limit where

$(\delta \rho) /(\rho)=\left(\rho^{\prime}-\rho\right) /(\rho) \ll 1$,

and

$(\delta K) /(K)=\left(K^{\prime}-K\right) /(K) \ll 1$,

where $\rho$ is the density of the medium and $\rho^{\prime}$ is the density of the scatterer, $K$ is the compressibility of the medium, and $K^{\prime}$ is the compressibility of the scatterer. The scattering amplitude is given by

$f(\theta)=\left(k^{2} a^{3}\right) /(3)[(\delta K) /(K)+(\delta \rho) /(\rho) \cos \theta] ;$

where $k$ is the wave vector of the insonifying wave. Following treatment by Jaynes, E. T. (personal communication), we approximate the scattering amplitude in terms of the phase velocity, $c=1 / \sqrt{K \rho}$, and the acoustic impedance, $Z=\rho c=\sqrt{\rho / K}$.

$(\delta K) /(K) \approx-(\delta Z) /(Z)-(\delta c) /(c)$, and

$(\delta \rho) /(\rho) \approx(\delta Z) /(Z)-(\delta c) /(c)$.

These expressions are obtained by using the binomial expansion and excluding terms of order two or higher in $(\delta \rho) /(\rho)$ and $(\delta K) /(K)$. Therefore,

$f(\theta) \approx-\left(k^{2} a^{3}\right) /(3)[(\delta Z) /(Z)(1-\cos \theta)+(\delta c) /(c)(1+\cos \theta)]$.

A local difference in acoustic impedance, or an impedance mismatch between the medium and the scatterer, therefore produces a scattering amplitude that has a backward cardioid shape. A local alteration in velocity produces a scattering amplitude that has a forward cardioid shape. Thus, for backscatter (i.e., $\theta=180^{\circ}$ ), only impedance mismatches $(\delta Z)$ contribute to the observed signals, and changes in impedance mismatch will result in a change in the energy of the backscattered wave.

\section{Appendix 2}

In this section we address the possibility that systematic changes in the attenuation of myocardial tissue throughout the physiologic cycle of contraction and relaxation might be responsible for the cyclic variation of integrated backscatter. Calculations are made for base-line attenuation in diastole with the use of published values for the attenuation of myocardium (53). Values for attenuation in systole are then derived after the assumption that the cyclic variation of backscatter is related strictly to variations in tissue attenuation rather than to alterations in intrinsic 
backscatter properties. We show that the change in attenuation required to produce the observed cyclic variation of backscatter represents an unrealistic possibility.

If we suppose that a change in attenuation represents the sole mechanism for the observed cyclic change in backscatter from both proximal and distal regions, then the necessary change in the attenuation can be estimated. For frequencies near $5 \mathrm{MHz}$ (the center frequency of our transducer), the attenuation of relaxed myocardium is $\sim 2.2 \mathrm{~dB} / \mathrm{cm}(53)$. Consequently, the attenuation of ultrasound through $6.3 \mathrm{~mm}$ of tissue (the round trip distance to and from the center of the proximal region) is $1.4 \mathrm{~dB}$. The cyclic change in IB from the proximal region is $5.5 \mathrm{~dB}$ (10). For this change in IB to result only from changes in the attenuation, the attenuation through $6.3 \mathrm{~mm}$ of contracted tissue must be $\sim 6.9 \mathrm{~dB}$ for $5 \mathrm{MHz}$ ultrasound. The resultant attenuation coefficient for contracted tissue would be $11 \mathrm{~dB} / \mathrm{cm}$, which represents an improbable increase to $500 \%$ of the base-line attenuation coefficient.

For the distal regions, the attenuation of relaxed myocardium for 5 $\mathrm{MHz}$ ultrasound through $10.8 \mathrm{~mm}$ of tissue (the round trip distance to and from the center of the distal region) is $\sim 2.4 \mathrm{~dB}$. The cyclic change in IB from the distal region is $\sim 7 \mathrm{~dB}(10)$. For this change in IB to occur only through changes in the attenuation, the magnitude of attenuation through $10.8 \mathrm{~mm}$ of contracted tissue must be $\sim 9.4 \mathrm{~dB}$ for $5 \mathrm{MHz}$ ultrasound. This would result in an attenuation coefficient of $8.7 \mathrm{~dB} /$ $\mathrm{cm}$ for contracted tissue, or an improbable increase to $400 \%$ of the baseline attenuation.

Although these increases are large enough to eliminate the consideration that changes in attenuation are solely responsible for changes in IB, we should also consider the possibility that a more modest change in attenuation is responsible for the difference in the amplitude of the cyclic variation of IB between proximal and distal regions. The magnitude of attenuation of $5 \mathrm{MHz}$ ultrasound through $4.5 \mathrm{~mm}$ of relaxed tissue (the round trip distance between the centers of the proximal and distal regions) is $\sim 1 \mathrm{~dB}$. The difference in the amplitude of the cyclic variation of IB between these two regions is $1.5 \mathrm{~dB}(10)$. In order for this regional difference in IB to occur only through changes in attenuation, the attenuation through $4.5 \mathrm{~mm}$ of contracted tissue must be $\sim 2.5 \mathrm{~dB}$. This would result in an attenuation coefficient of $5.6 \mathrm{~dB} / \mathrm{cm}$ for contracted tissue, which represents an unlikely increase to $250 \%$ of the baseline attenuation.

\section{Acknowledgments}

We appreciate the expert technical assistance of Delbert McGraw.

This work was supported in part by National Institutes of Health grant HL17646, by Specialized Centers of Research in Ischemic Heart Disease, National Aeronautics and Space Administration grant NSG1601, and by a Squibb Cardiovascular Research Fellowship (to Dr. Perez).

\section{References}

1. Perez, J. E., E. I. Madaras, B. E. Sobel, and J. G. Miller. 1984. Quantitative myocardial characterization with ultrasound. Automedica (Lond.). 5:201-218.

2. Miller, J. G., J. E. Perez, and B. E. Sobel. 1985. Ultrasonic characterization of myocardium. Prog. Cardiovasc. Dis. 28:85-110.

3. Mimbs, J. W., D. Bauwens, R. D. Cohen, M. O’Donnell, J. G. Miller, and B. E. Sobel. 1981. Effects of myocardial ischemia on quantitative ultrasonic backscatter and identification of responsible determinants. Circ. Res. 49:89-96.

4. Mimbs, J. W., M. O’Donnell, J. G. Miller, and B. E. Sobel. 1981. Detection of cardiomyopathic changes induced by doxorubicin based on quantitative analysis of ultrasonic backscatter. Am. J. Cardiol. 47: 1056-1060.

5. Miller, J. G., J. E. Perez, E. I. Madaras, J. G. Mottley, P. H. Johnston, E. D. Blodgett, L. J. Thomas III, and B. E. Sobel. 1983. Myocardial tissue characterization: an approach based on quantitative back- scatter and attenuation. Proc. 1983 IEEE Ultrasonics Symp., Vol. 83CH1947-1. 782-793.

6. Barzilai, B., E. I. Madaras, B. E. Sobel, J. G. Miller, and J. E. Perez. 1984. Effects of myocardial contraction on ultrasonic backscatter before and after ischemia. Am. J. Physiol. 247:H478-H483.

7. Perez, J. E., B. Barzilai, E. I. Madaras, R. M. Glueck, J. E. Saffitz, P. H. Johnston, J. G. Miller, and B. E. Sobel. 1984. Application of ultrasonic tissue characterization for longitudinal assessment and differentiation of calcification and fibrosis in cardiomyopathy. J. Am. Coll. Cardiol. 4:88-95.

8. Madaras, E. I., B. Barzilai, J. E. Perez, B. E. Sobel, and J. G. Miller. 1983. Changes in myocardial backscatter throughout the cardiac cycle. Ultrasonic Imaging. 5:229-239.

9. Thomas, L. J. III, S. A. Wickline, J. E. Perez, B. E. Sobel, and J. G. Miller. 1985. Ultrasonic tissue characterization by analysis of integrated backscatter in real-time. IEEE Trans. Ultrasonics, Ferroelectrics, and Frequency Control. In press.

10. Wickline, S. A., L. J. Thomas III, J. G. Miller, B. E. Sobel, and J. E. Perez. 1985. The dependence of myocardial ultrasonic backscatter on contractile performance. Circulation. 72:183-192.

11. Olshansky, B., S. M. Collins, and D. J. Skorton. 1984. Variation of left ventricular myocardial gray level on two-dimensional echocardiograms as a result of cardiac contraction. Circulation. 70:972-977.

12. Spotnitz, H. M., E. H. Sonnenblick, and D. Spiro. 1966. Relation of ultrastructure to function in the intact heart: sarcomere structure relative to pressure volume curves of intact left ventricles of dog and cat. Circ. Res. 18:49-66.

13. Laks, M. M., M. J. Nisenson, and H. J. C. Swan. 1967. Myocardial cell and sarcomere lengths in the normal dog heart. Circ. Res. 21:671678.

14. Yoran, C., J. W. Covell, and J. R. Ross. 1973. Structural basis for the ascending limb of left ventricular function. Circ. Res. 32:297303.

15. LeWinter, M. M., R. S. Kent, J. M. Kroener, T. E. Carew, and J. W. Covell. 1975. Regional differences in myocardial performance in the left ventricle of the dog. Circ. Res. 37:191-199.

16. Sabbah, H. N., M. Marzilli, and P. D. Stein. 1981. The relative role of subendocardium and subepicardium in left ventricular mechanics. Am. J. Physiol. 240:H920-H926.

17. Gallagher, K. P., G. Osakada, O. M. Hess, J. A. Koziol, S. Kemper, and J. R. Ross, Jr. 1982. Subepicardial segmental function during coronary stenosis and the role of myocardial fiber orientation. Circ. Res. 50:352-359.

18. Stein, P. D., M. Marzilli, H. N. Sabbah, and T. Lee. 1980. Systolic and diastolic pressure gradients within the left ventricular wall. $\mathrm{Am}$. J. Physiol. 238:H625-H630.

19. Kirk, E. S., and C. R. Honig. 1964. Nonuniform distribution of blood flow and gradients of oxygen tension within the heart. Am. J. Physiol. 207:661-668.

20. Huisman, R. M., G. Elzinga, N. Westerhof, and P. Sipkema. 1980. Measurement of left ventricular wall stress. Cardiovas. Res. 14: 142-153.

21. Streeter, D. D., Jr., and W. T. Hanna. 1973. Engineering mechanics for successive states in canine left ventricular myocardium. II. Fiber angle and sarcomere length. Circ. Res. 33:656-664.

22. Streeter, D. D., R. N. Vaishnav, D. J. Patell, H. M. Spotnitz, J. Ross, Jr., and E. H. Sonnenblick. 1970. Stress distribution in the canine left ventricle during systole and diastole. Biophys. J. 10:345-363.

23. Chadwick, R. S. 1982. Mechanics of the left ventricle. Biophys. J. 39:279-288.

24. Ghista, D. N., and H. Sandler. 1969. An analytic elastic-viscoelastic model for the shape and the forces in the left ventricle. J. Biomech. 2:35-47.

25. Pierce, W. H. 1981. Body forces and pressures in elastic models of the myocardium. Biophys. J. 34:35-59.

26. Shung, K. K., and J. M. Reid. 1977. Ultrasonic scattering from 
tissue. Proc. 1977 Ultrasonics Symp., IEEE Cat. 77CH12364-ISU. 230232.

27. O’Donnell, M., J. W. Mimbs, and J. G. Miller. 1981. Relationship between collagen and ultrasonic backscatter in myocardial tissue. $J$. Acoust. Soc. Am. 69:580-588.

28. Mimbs, J. W., M. O’Donnell, D. Bauwens, J. G. Miller, and B. E. Sobel. 1980. The dependence of ultrasonic attenuation and backscatter on collagen content in dog and rabbit hearts. Circ. Res. 47:4958.

29. Caulfield, J. B., and T. K. Borg. 1979. The collagen network of the heart. Lab. Invest. 40:364-372.

30. Orenstein, J., D. Hogan, and S. Bloom. 1980. Surface cables of cardiac myocytes. J. Mol. Cell. Cardiol. 12:771-780.

31. Fabiato, A., and F. Fabiato. 1978. Myofilament-generated tension oscillations during partial calcium activation and activation dependence of the sarcomere length-tension relation of skinned cardiac cells. J. Gen. Physiol. 72:667-699.

32. Lazarides, E. 1980. Intermediate filaments as mechanical integrators of cellular space. Nature (Lond.). 283:249-256.

33. Price, M. G. 1984. Molecular analysis of intermediate filament cytoskeleton-a putative load-bearing structure. Am. J. Physiol. 246: H566-H572.

34. Wang, K. 1982. Myofilamentous and myofibrillar connections: role of titin, nebulin, and intermediate filaments. In Muscle Development: Molecular and Cellular Control. M. L. Pearson and H. F. Epstein, editors. Cold Spring Harbor Laboratory, New York. 439-452.

35. Braunwald, E., J. Ross, Jr., and E. H. Sonnenblick. 1976. Mechanics of Contraction of the Normal and Failing Heart. Little, Brown, and Co., Boston. Second ed. 1-417.

36. Sonnenblick, E. H. 1964. Series elastic and contractile elements in heart muscle: change in muscle length. Am. J. Physiol. 207:13301338.

37. Brady, A. J., S. T. Tan, and N. V. Ricchiuti. 1981. Perturbation measurements of papillary muscle elasticity. Am. J. Physiol. 241:H155H173.

38. Fish, D., J. Orenstein, and S. Bloom. 1984. Passive stiffness of isolated cardiac and skeletal myocytes in the hamster. Circ. Res. 54:267276.
39. Yin, F. C. P. 1981. Ventricular wall stess. Circ. Res. 49:829-842. 40. Fung, Y. C. 1981. Biomechanics. Mechanical Properties of Living Tissues. Springer-Verlag, New York. 38-53, 340-342.

41. Snedecor, G. W., and W. G. Cochran. 1980. Statistical Methods. Iowa State University Press, Ames. Seventh ed. 1-505.

42. Jennings, R. B., and C. E. Ganote. 1974. Structural changes in myocardium during acute ischemia. Circ. Res. 34/35(Suppl. III):156172.

43. Kloner, R. A., C. E. Ganote, D. A. Whalen, and R. B. Jennings. 1974. Effect of a transient period of ischemia on myocardial cells. Am. J. Pathol. 74:399-422.

44. Mirsky, I. 1969. Left ventricular stress in the intact human heart. Biophys. J. 9:189-208.

45. Feit, T. S. 1979. Diastolic pressure-volume relations and distribution of pressure and fiber extension across the wall of a model left ventricle. Biophys. J. 28:143-166.

46. Moriarty, T. F. 1980. The law of Laplace: its limitations as a relation for diastolic pressure, volume, or wall stress of the left ventricle. Circ. Res. 46:321-331.

47. Mottley, J. G., and J. G. Miller. 1982. Anisotropy of ultrasonic attenuation in canine heart and liver. Ultrasonic Imaging. 4:180. (Abstr.)

48. Sasayama, S., D. Franklin, J. R. Ross, Jr., W. S. Kemper, and D. McKown. 1976. Dynamic changes in left ventricular wall thickness and their use in analyzing cardiac function in the conscious dog. Am. J. Cardiol. 38:870-879.

49. Glueck, R. M., J. G. Mottley, J. E. Perez, J. G. Miller, and B. E. Sobel. 1985. Systematic changes in quantitative ultrasonic indexes caused by the contractile state of muscle. Circ. Res. 56:683-689.

50. Huxley, A. F. 1974. Muscular contraction. J. Physiol. 243:1-43.

51. Mason, P. 1978. Dynamic stiffness and crossbridge action in muscle. Biophys. Struct. Mech. 4:15-25.

52. Morse, P. M., and K. V. Ingard. 1968. Theoretical Acoustics. McGraw-Hill Book Co., New York. 411-414.

53. O'Donnell, M., J. W. Mimbs, and J. G. Miller. 1979. The relationship between collagen and ultrasonic attenuation in myocardial tissue. J. Acoust. Soc. Am. 65:512-517. 